\title{
Review
}

\section{Why not just switch on the light?: light and its versatile applications in the field of nanomedicine}

\author{
Roman Lehner ${ }^{1}$ and Patrick Hunziker ${ }^{1,2, *}$ \\ ${ }^{1}$ University Hospital Basel, Intensive Care Unit, \\ Petersgraben 4, 4031 Basel, Switzerland \\ ${ }^{2}$ CLINAM Foundation for Nanomedicine, Intensive Care \\ Unit, Petersgraben 4, 4031 Basel, Switzerland
}

\begin{abstract}
Over the last decade, the emerging field of nanomedicine has undergone rapid progresses. Different internal and external stimuli like $\mathrm{pH}$, temperature, radiation, ultrasound or light have been introduced to expand the diagnostic and therapeutic options of various applications within the field. This review focuses on the novel application of light in the field of nanomedicine as a mechanism to control drug delivery, release and biochemical and genetic functionality at the target. The field of functional nanomaterials for medicine, and in particular of light responsive nanocarriers, polymers and biomolecules offer new therapeutic options but also requires substantial further research to render this approach broadly applicable in clinical practice.
\end{abstract}

Keywords: light; nanoparticles; photodynamic therapy; photothermal therapy.

\section{Introduction}

A variety of new nanomaterials such as polymers, liposomes, micelles, dendrimers or metallic nanoparticles have shaped the constant and rapid progressing field of nanomedicine within the last decade. Today, nanoparticles have found their way into the clinical domain as drug delivery systems, for imaging, sensing and therapy. To provide specific characteristics, nanoparticles can be tailored into "intelligent" nanoparticles through stimulus responsiveness. Stimuli responsive nanoparticle for medicine can be classified based on the type of stimuli into locally/internal triggered systems responding to their close environment, and externally triggered stimuliresponsive nanoparticles that can be remote-controlled even from outside the body. Various internal stimuli such as $\mathrm{pH}$, redox potential, enzymatic activity, temperature and external stimuli like ultrasound, magnetic field, temperature and light

*Corresponding author: Patrick Hunziker, University Hospital Basel, Intensive Care Unit, Petersgraben 4, 4031 Basel, Switzerland E-mail: Patrick.Hunziker@swissnano.org

Previously published online December 20, 2012 are being intensively investigated. Out of all these stimuli, light shows particularly attractive features such as high sensitivity, ease of controllability and a range of physical properties (e.g., light intensity, wavelength, exposure time) that allow in principle to design selective and multiplexed activities to be programmed into a material. Therefore, it is not surprising that a significant effort is currently invested into the development of light responsive nanoparticles, oligonucleotides or peptides.

This review presents a brief overview on light and its applications within the field of nanomedicine. It will describe mechanisms of light-controlled drug delivery, controlled drug release, light-controlled activity switching for biochemical mechanisms, gen expression and gene silencing at the target. The aim of this paper is to identify opportunities, describe gaps, and thus to stimulate further research, such that light- controlled nanomedical therapies develop into well tolerated, highly effective interventions to the benefit of the patient.

\section{Application of light in nanomedicine}

Light for triggered release and activation of drugs and biomolecules

Despite the efforts in drug delivery design and developments, major obstacles such as endosomal escape and efficient payload release within the diseased tissue and cell have to be overcome for efficient clinical application. Light can be used to enhance drug delivery and payload release by applying light sensitive moieties to drug delivery platforms and of photolabile protecting groups to biologically active molecules by a strategy called caging (Figure 1).

Caging is an attractive way of turning biological molecules e.g., nucleic acids (DNA, RNA), proteins or peptides light sensitive for the investigation of biological processes. Caged biomolecules incorporate a light-removable protecting group, so-called "caging group", which aborts its native biological or biochemical activity. Since caging of ATP was first reported in 1978 by Kaplan et al., several different photolabile groups have been introduced to turn biomolecules temporarily inactive (1-3). Examples of caged biomolecules are neurotransmitters (4), nucleotides (4), peptides (5, 6), siRNA (7) or DNA (8). The most widely used caged neurotransmitter so far is glutamate for which different protecting groups have been applied (9). RNA interference is a mechanism able to inhibit protein translation by gene silencing. Nguyen et al. caged a 1-(2-nirophenyl)ethyl (NPE) group to the $5^{\prime}$ terminal phosphate of the siRNA antisense strand, which inactivates 
$\mathbf{A}$

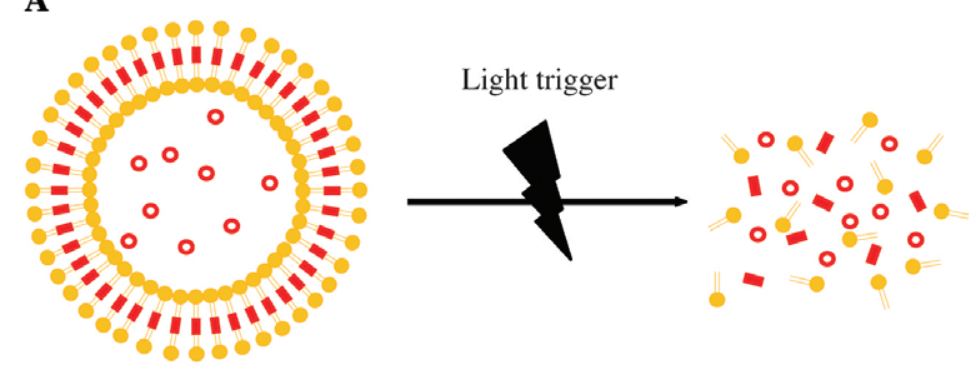

Light sensitive moiety

- Payload

B

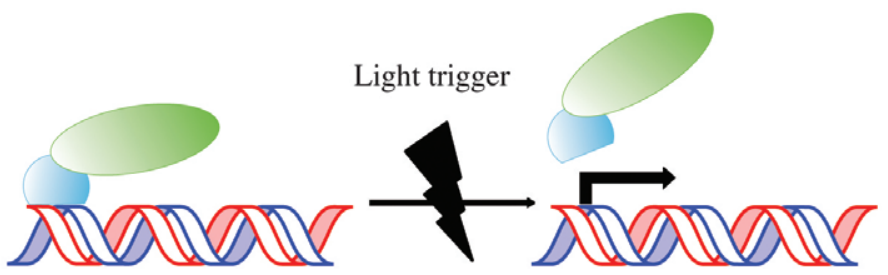

Figure 1 (A) Light responsive drug delivery system built through covalent incorporation of specific light-sensitive chemical groups (red rectangles) with the aim to locally release cargo (red circles) by illumination. (B) Site-specific caging of DNA can be used for light-activated gene expression.

the siRNA activity (10). They could demonstrate an approximately $70 \%$ efficient light induced RNA interference using wavelengths between 345 and $385 \mathrm{~nm}$. An alternative form to siRNA mediated control of gene silencing has been reported by Young et al. (11). They introduced a caging group to DNAzymes to inhibit hybridization with mRNA. DNAzymes are enzymatically active desoxyoligonucleotides, which can cleave RNA in a site-specific manner. Translation of mRNA can be aborted upon illumination with UV-light to photorelease the caging group. Caging of DNA has widely been studied as seen from several publications $(8,12,13)$. To render those approaches suitable for future clinical application, extension of the work towards longer wavelengths and therefore reduced toxicity should be accompanied by identification of suitable in vitro and in vivo disease models of human disease.

Light-responsive materials for drug delivery can be constructed by the covalent incorporation of specific light-sensitive chemical groups with the aim to locally release cargo by illumination. The synthesis of a photocleavable amphiphilic block copolymer has been demonstrated by Cabane et al. (14). As photosensitive molecule they introduced an o-nitrobenzyl linker between the hydrophobic and hydrophilic blocks, which form vesicles or micelles upon self-assembly in aqueous solution. Successful disruption of the vesicles could be demonstrated after irradiation with UV-light by electron microscopy and dynamic lights scattering data. The design of photocleavable liposomes for drug delivery using different photolabile groups has been reported in several publications $(15,16)$. Dvir et al. presented a simple proof of concept by carboxylated polystyrene nanoparticles labeled with the unspecific amino acid sequence YIGSR, which adheres to $\beta 1$ integrins present on most cell surfaces (4, 17). The peptide was caged with a nitrobenzyl group, which could be removed via illumination, leading to nanoparticle binding to the cells. Another approach of light sensitive nanoparticles currently being investigated uses nano-impellers. Nano-impellers are nanomechanical systems allowing the spatiotemporal drug release upon illumination, turning them into an attractive application for clinical trials $(5,6$, $18,19)$. A clear disadvantage of many published systems is the requirement for light energy in the UV range, limiting their application due to phototoxicity and the very limited penetration range of short wavelength light in biological tissues.

\section{Light induced gene expression and control of gene silencing}

Light-mediated control of gene expression and silencing is a powerful and fast growing field in the areas of systems biology, functional genomics and biotechnology. Spatiotemporal and precise gene expression represents the most fundamental level of further complex biological processes such as the control of thousand of proteins and the associated control of metabolic processes. Therefore, light represents a suitable stimulus for in vitro as well as in vivo studies as it is non-invasive, sensitive and allows the spatiotemporal and precise application without interfering with metabolic conditions. Light-induced gene expression can either be achieved using caged biomolecules such as plasmid DNA $(12,13)$, transcription factors $(8,20,21)$ or via photoreceptors harboring a chromophore $(9,22,23)$. Several reports focused on caged plasmid DNA's have been published, whereas effective gene 
expression remained a major challenge due to ineffective random backbone modifications $(8,10)$. In addition, successful uncaging and activation of gene expression required high levels of light that can cause phototoxicity (24). A more effective approach for light controlled activation of gene expression was shown by Yamaguchi et al. using a site-specific labeling of the promoter region with a biotinylated photolabile group, leading to effective activation of gene expression in HeLa cells even under low levels of light (12). A successful gene regulation system combining light-sensitive proteins and programmable zinc finger transcription factors has been published by Polstein et al. (14, 20 ). The system is based on two light-inducible fusion proteins from Arabidopsis thaliana, GIGANTEA (GI) fused to a Zinc finger protein leading the complex to the target DNA sequence and the LOV domain of FKF1 fused to the transcriptional activation domain VP16. Illumination with light leads to fusion of the GI and LOV domain, which guides the LOV-VP16 domain to the target gene and enables gene expression.

Beside light induced gene expression, the focus of photochemical control of gene function has been directed to RNA interference. RNA interference represents one of the major approaches leading to gene silencing/such as that occurring in embryogenesis) and is being extensively explored as a therapeutic strategy for different kind of diseases, including cancer. Two primary approaches for photochemical regulation have been developed. The caging groups are either covalently attached to the phosphate backbone or terminal phosphates or on the nucleotide bases to inhibit the further process of RNA induced silencing (Figure 2). The first report of caged siRNA has been described by Shah et al. using 1-(4, 5-dimethoxy-2nitrophenyl)ethyl (DMNPE) attached to the phosphate backbone which only showed a $3 \%$ caging efficiency $(15,16,24)$. Caging of guanosine and thymidin bases by attaching 2-(2nitrophenyl)propy (NPP) groups has been reported by Mikat and Heckel (25). The modifications have shown knockdown efficiency of about $75 \%$ after light irradiation. Jain et al. designed a siRNA caged at the terminal phosphates with a cyclo-dodecyl DMNPE, which is more bulky and therefore shows higher steric hindrance $(11,26)$. In contrast to the DMNPE, which has been introduced to the phosphate backbone, siRNA terminally caged with cyclo-dodecyl DMNPE showed an efficiency of $89 \%$.

\section{Photodynamic therapy}

The therapeutic effect of light has been known for thousands of years and was applied by the Egyptians, Indians and Chinese $(8,12,13,27)$. Its therapeutic relevance to cancer treatment and further development into the photodynamic therapy (PDT) was reported at the beginning of the last century by Oscar Raab, a German medical student and his professor Hermann von Tappeiner (28). The principle of photodynamic therapy involves the administration of a photosensitizer, which will form highly reactive singlet oxygen radical (ROS) from molecular oxygen after illumination with light (Figure 3). Singlet oxygen radicals are known to cause severe damage to biological macromolecules such as membrane lipids and proteins (29). After absorption of light, photosensitizers will change from a ground state into a relatively long-lived excited triple state and a short-lived excited single state. The excited single state can return to the ground state by emitting fluorescence that can be used for clinical detection. In the excited triple state, the photosensitizer molecule can transfer its energy via a type-I or -II reaction. In the type-I reaction, the photosensitizer can react directly with a surrounding substrate to form radicals, which then can further interact with oxygen to produce oxygenated products. In the type-II reaction the energy of the excited photosensitizer can be directly transferred to oxygen to form highly reactive singlet oxygen (30).

Photodynamic therapy has found its way into clinical applications using nanocarrier platforms as delivery system such as photodynamic eye therapy for the treatment of neovascularization, abnormal endothelial proliferation or for different cancer treatments (bladder, skin, head and neck, esophageal, or endobronchial cancer) $(31,32)$. A number of nanoparticle-
A

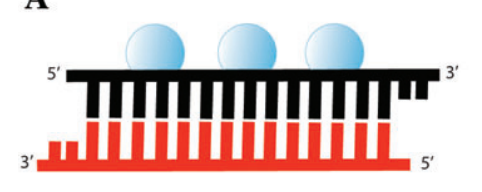

B

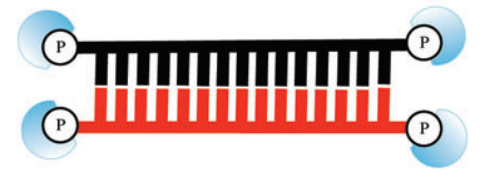

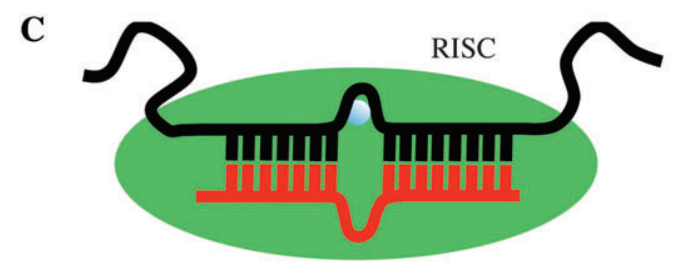

Figure 2 Schematic illustration of caged siRNA strategies. (A) Caged phosphate backbone and (B) caged terminal phosphates of siRNA. (C) Introduction of a caged base into a siRNA antisense strand to inhibit RNA interference. 


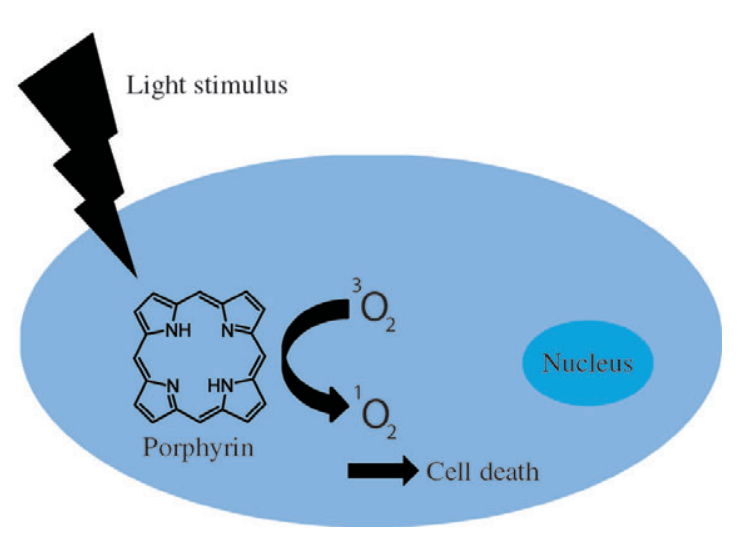

Figure 3 Light activation of a photosensitizer leads to the formation of highly reactive singlet oxygen for selective cell killing.

based photodynamic therapies have been approved by the U.S. Food and Drug Administration (FDA) such as e.g., Visudyne $^{\circledast}$, Photofrin ${ }^{\circledast}$, Levulan ${ }^{\circledast}$ Kerastick $^{\circledast}$ opening doors for future applications and new possible approaches for future therapies (31). There are several advantages of PDT as a clinical application including a single dose requirement for treatment followed by illumination compared to radiotherapy and chemotherapy, which both depend on a treatment over several weeks or months. Further, it is a local treatment without interfering with the whole organism and retreatment can be simply done in the case of recurrence of a tumor without severe healthy tissue damage. However, further development in the direction of controlled drug release, as well as improved payload capacity of nanoparticle-based delivery systems is warranted.

\section{Photochemical internalization (PCI)}

One of the key challenges that still needs to be overcome in order to enable the clinical application of therapeutic delivery of different payloads is endosomal escape. Various strategies have been developed to achieve endosomal escape and these are either based on the characteristic endosomal property of a lower intracellular $\mathrm{pH}$ compared to the cytoplasm, incorporation of fusogenic peptides into the endosomal membrane or a strategy called photochemical internalization (PCI). PCI is a site-specific method for intracellular drug delivery by induced endolysosomal escape based on photostimulation. The principle behind PCI relies on photodynamic therapy targeted to endosomes or lysosomes, whereas the vesicular membrane bursts after coming into contact with highly reactive singlet oxygen after illumination of the photosensitizer (Figure 4). In comparison to conventional photodynamic therapy, where the intracellular localization of the photosensitizer does not play an important role because of its complete cellular destruction, PCI is based on the specific accumulation of the photosensitizer in the endolysosomal compartment to achieve endosomal escape without harming the rest of the cell (33).

A fate that may be a consequence to nanocarriers after endocytotic uptake, is the accumulation in the endolysosome, whereas PCI offers a good solution. Lai et al. have demon-

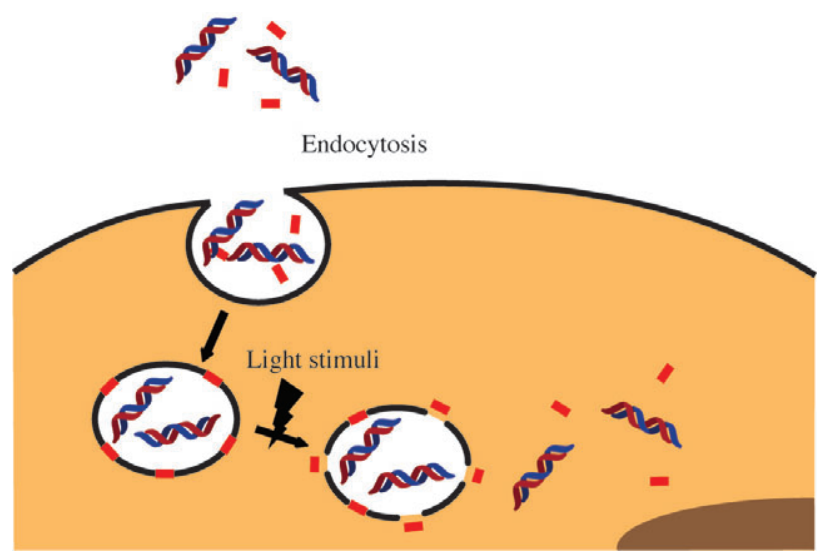

Figure 4 Intracellular drug delivery induced by endolysosomal escape based on photostimulation. Light-irradiation after endocytotic uptake leads to endolysosomal membrane burst upon highly reactive singlet oxygen.

strated the effective delivery of doxorubicin and saporin by photochemical internalization using a poylamidoamine (PAMAM) dendrimer $(34,35)$. Recently, Lu et al. reported the overcoming of doxorubicin drug resistance in vivo by applying dendrimer phtalocyanine-encapsulated polymeric micelles combined with doxorubicin into doxorubicin-resistant bearing mice (36). It has also been shown by Nishiyama et al. that PCI can mediate gene transfection, using a combinational system including polymeric micelles incorporating pDNA and a dendrimer-based photosensitizer (37). Both polymeric micelles are assumed to be taken up by the cells at the same time. After illumination, a remarkable enhancement of transgene expression could be detected while retaining cell viability. Beside enhancement of gene expression, PCI can also be used for siRNA mediated gene knockdown studies. The first application of PCI to facilitate endosomal escape of siRNA was reported in 2007 by Oliveira et al. (38). They used TPPS $_{2 \mathrm{a}}$ as photosensitizer together with a siRNA able to knockdown epidermal growth factor receptor (EGFR) expression. Complexes of EGFR siRNA and Lipofectamine were applied to the cells. A 10-fold increased efficiency in EGFR knockdown could be detected after illumination compared to siRNA treatment alone. A recently published study by Varkouhi et al. presents PCI mediated enhancement of gene silencing using a polymer-based nanocarrier platform consisting out of cationic polymethacrylates and N,N,N-trimethylated chitosan (39). Furthermore, PCI can enhance the effect of targeted protein toxins that have reached the tumors cells (40). Targeted protein toxins consist of a protein toxin moiety, initiating cytotoxicity and a cell binding moiety, which targets the protein actively to the cell. Denileukin diftitox is the first FDA approved protein toxin for treatment of cutaneous T-cell lymphoma.

\section{Photothermal therapy}

Hyperthermia is a non-invasive approach for cancer treatment based on the principle of spatiotemporally increasing the temperature to promote selective destruction of cancer cells, which 
are more sensitive to hyperthermia than normal cells due to their higher metabolic rates. Several different approaches have already been applied for delivery of thermal energy such as ultrasound, microwaves or radiofrequency pulses (41-43). A disadvantage is their dispersive property with the result that high fluences (high amount of particles that intersect an area at a specific timepoint) are needed, which lead to undesirable hyperthermic effects on surrounding tissues. Within the last few years, gold nanoparticles have received increasing attention due to their versatile applications such as imaging, cancer therapy, drug delivery and especially because of their unique surface plasmon resonance (SPR) absorption at visible or Near-infrared (NIR) wavelengths (44). The use of NIR is desirable due to its deep penetrating capacity and minimal interference with water and biomolecules in tissues. The principle of photothermal therapy is the combination of light and gold nanoparticles (e.g., gold nanospheres, nanorods, nanoshells, nanocages) for clinical treatment. Illumination of gold nanoparticles leads to conversion of absorbed light into thermal energy, the resulting heat causes cell and tissue destruction (Figure 5). El-Sayed et al. have shown the use of gold nanorods labeled with an anti-EGFR antibody for selective photothermal treatment of cancer cells (45). A dual-modality approach for photodynamic and photothermal therapy has been recently published by Kuo et al. (46). They used gold nanomaterials conjugated with the hydrophilic photosensitizer, indocyanine green, to achieve photothermal therapy (PTT) and photodynamic therapy (PDT). The combination of PTT and PDT showed enhanced destruction of cancer cells in contrast to their single application effectiveness. Photothermal tumor ablation in mice could be proven by O'Neal et al. using gold nanoshells (47). They subcutaneously injected murine colon carcinoma cells into immune-competent mice, followed by injection of gold nanoshells. After $6 \mathrm{~h}$ of circulation, tumors were illuminated with NIR. All treated mice looked healthy and tumor free after more then 90 days post-treatment.

\section{Photoswitchable fluorescent nanoparticles}

Over the past decades a huge number of nanoparticles made of different materials have been developed and these have biological and medical applications. Whereas many of those platforms have been developed for the purpose of improved drug delivery and therapy another promising direction, which has attracted considerable interest is molecular imaging. Nanoparticle-based imaging offers a non-invasive and quantitative detection method of biomolecules, while at the same time improves sensitivity and specificity of diagnostic imaging as a tool for e.g., early cancer detection. Fluorescence spectroscopy is a powerful method used for molecular imaging of living cells, allowing very sensitive measurements at high resolution. Fluorescence imaging is based on the principle of the absorption of light by a fluorescent dye (e.g., fluorophore or fluorochrome), which emits fluorescent light at a longer wavelength than that absorbed. Fluorescent nanoparticles such as polymer NPs, silica NPs, gold NPs or quantum dots (QD) gained intensive interest during the last years. They can be produced by doping the material with suitable fluorescent dyes or luminescent metals while quantum dots can directly be applied due to their intrinsic fluorescence properties (48). The advantages of fluorescent nanoparticles compared to normal organic dyes are higher brightness due to the fact that a nanoparticle can carry several dye molecules, increase in photostability because the dyes are entrapped within the nanoparticles, higher specificity upon their functionalization properties and their long-term-tracking ability.

Understanding cellular networks is the essential key factor to understand the complex structure of certain diseases. To achieve this goal, significant progress has been made in the development of quantum dots for cellular sensing which have been recently reviewed (49). Sensing quantum dots are based on the principle of the recognition of an analyte, which acts as a fluorescence quencher, by a receptor or chemosensor causing changes upon emission of the fluorophore. Various quantum dots based on overcoating of the core with $\mathrm{ZnS}$ or $\mathrm{CdSe}$ to improve their fluorescence quantum yield and additional modification of the surface properties to increase their emission have been reported (50-52). Furthermore, this concept can be used to prepare glucose or maltose sensing systems, whereas a photoinduced electron transfer (PET) from the coating molecules to the valence band of an excited quantum dot results in emission quenching as shown by Cordes and Sandros et al. $(53,54)$.

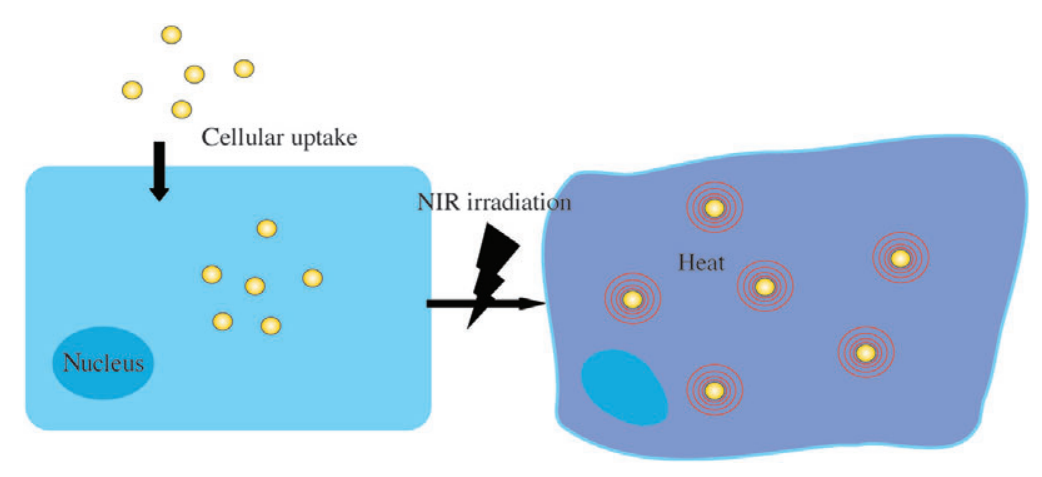

Figure 5 Photothermal therapy is based on intracellular uptake of gold nanoparticles, which after irradiation with near-infrared light convert absorbed light into thermal energy for specific destruction of cancer cells. 
Beside the mentioned applications, the most largely exploited photoswitchable fluorescent sensing mechanism is by fluorescence resonance energy transfer (FRET) by which an energy transfer from a QD to a fluorophore will be determined. Until now, several strategies allowing generic on/off photoswitching based on FRET have been reported (55-57). Another more complex approach for photoswitching has been explored by dye doped nanoparticles allowing coactive triggering of multiple processes. These dye doped sensing nanoparticles were first introduced by Kopelman in the late 1990s and are called photonic explorers for bioanalysis with biologically localized embedding (PEBBLEs) (58). The insertion of chemosensors into nanoparticles shows several advantages as minimization of interaction with other biomolecules within the cells or the introduction of multifunctional sensing schemes as for example by $\mathrm{pH}$ sensitivity. A wide variety of PEBBLEs have been reported since their development, whereas further literature can be found here (59-61).

However, the ability of QDs to combine molecular imaging and therapy can open new doors for clinical application, but the toxicity of especially heavy metals used in QD synthesis such as cadmium is an important concern (62).

\section{Limiting factors of light}

Light as external stimuli for enhanced drug delivery, cargo release, imaging and therapy offers some attractive features such as high sensitivity and spatiotemporal control. However, the major drawback of light is tissue penetration depth, restricting its applications. Solutions to overcome this problem have been made by development of near infrared (NIR) light sensitive photochemical compounds. Near-infrared light at wavelengths of 700-1000 nm can penetrate up to several centimeters deep into tissues without causing any damage (63). This renders NIR much more attractive than the often used UV-light regarding its potential for severe tissue damage. The use of two-photon excitation systems as well as application of upconverting nanoparticles, both provide possible solutions of how to overcome the problem of tissue penetration depth. Two-photon excitation depends on the principle of exciting a caged group by absorption of two photons induced via a pulsed laser. This method allows the usage of caged groups, which absorb light in the UV range but can be excited via pulsed NIR. Upconverted nanomaterials are able to convert NIR into UV light, which generates the same benefit as seen for the two-photon excitation. Practical application of such systems into clinical trials might still need some time due to the fact that most published data using NIR, show irradiation times of hours, which might interfere with clinical practice (64).

\section{Conclusion}

The use of light as an external stimulus is a promising approach for a wide range of applications within the field of nanomedicine based on its attractive properties such as sensitivity and biocompatibility, in particular for wavelengths longer than UV. Furthermore, it has shown advantages regarding its high spatial and temporal precision. However, the major drawback of light is tissue penetration depth, which severely restricts the applications of caged compounds, light sensitive drug delivery systems and light-based therapies into clinical application. Thus, approaches like the usage of NIR linked to two-photon uncaging and up-converting systems seem to be promising but further optimization of these methods is needed to increase the chance of further application in clinical trials.

\section{References}

1. Kaplan JH, Forbush B, Hoffman JF. Rapid photolytic release of adenosine 5'-triphosphate from a protected analogue: utilization by the Na:K pump of human red blood cell ghosts. Biochemistry 1978;17:1929-35.

2. Pelliccioli AP, Wirz J. Photoremovable protecting groups: reaction mechanisms and applications. Photochem Photobiol Sci 2002;1:441-58.

3. Han G, Mokari T, Ajo-Franklin C, Cohen BE. Caged quantum dots. J Am Chem Soc 2008;130:15811-3.

4. Walker JW, Reid GP, McCray JA, Trentham DR. Photolabile 1-(2-nitrophenyl)ethyl phosphate esters of adenine nucleotide analogs. Synthesis and mechanism of photolysis. J Am Chem Soc 1988;110:7170-7.

5. Rothman DM, Petersson EJ, Vázquez ME, Brandt GS, Dougherty DA, Imperiali B. Caged Phosphoproteins. J Am Chem Soc 2005; 127:846-7.

6. Lawrence DS. The preparation and in vivo applications of caged peptides and proteins. Curr Opin Chem Biol 2005;9:570-5.

7. Matsushita-Ishiodori Y, Ohtsuki T. Photoinduced RNA interference. Acc Chem Res 2012;45:1039-47.

8. Ceo LM, Koh JT. Photocaged DNA provides new levels of transcription control. ChemBioChem 2012;13:511-3.

9. Ellis-Davies GCR. Caged compounds: photorelease technology for control of cellular chemistry and physiology. Nat Meth 2007;4:619-28.

10. Nguyen QN, Chavli RV, Marques JT, Conrad PG, Wang D, He W, et al. Light controllable siRNAs regulate gene suppression and phenotypes in cells. BBA - Biomembranes 2006;1758:394-403.

11. Young DD, Lively MO, Deiters A. Activation and deactivation of DNAzyme and antisense function with light for the photochemical regulation of gene expression in mammalian cells. $\mathrm{J}$ Am Chem Soc 2010;132:6183-93.

12. Yamaguchi S, Chen Y, Nakajima S, Furuta T, Nagamune T. Light-activated gene expression from site-specific caged DNA with a biotinylated photolabile protection group. Chem Commun 2010;46:2244-6.

13. Monroe WT, McQuain MM, Chang MS, Alexander JS, Haselton FR. Targeting expression with light using caged DNA. J Biol Chem 1999;274:20895-900.

14. Cabane E, Malinova V, Meier W. Synthesis of photocleavable amphiphilic block copolymers: toward the design of photosensitive nanocarriers. Macromol Chem Phys 2010;211:1847-56.

15. Chandra B, Subramaniam R, Mallik S, Srivastava DK. Formulation of photocleavable liposomes and the mechanism of their content release. Org Biomol Chem 2006;4:1730-40.

16. Yavlovich A, Smith B, Gupta K, Blumenthal R, Puri A. Lightsensitive lipid-based nanoparticles for drug delivery: design principles and future considerations for biological applications. Mol Membr Biol 2010;27:364-81. 
17. Dvir T, Banghart MR, Timko BP, Langer R, Kohane DS. Phototargeted nanoparticles. Nano Lett 2010;10:250-4.

18. Lu J, Choi E, Tamanoi F, Zink JI. Light-activated nanoimpellercontrolled drug release in cancer cells. Small 2008;4:421-6.

19. Lau YA, Ferris DP, Zink JI. Proceedings of SPIE. Nanoscale Imaging, Sensing, and Actuation for Biomedical Applications VII, SPIE; 2010.

20. Polstein LRL, Gersbach CAC. Light-inducible spatiotemporal control of gene activation by customizable zinc finger transcription factors. J Am Chem Soc 2012;134:16480-3.

21. Govan JMJ, Lively MOM, Deiters AA. Photochemical control of DNA decoy function enables precise regulation of nuclear factor кB activity. J Am Chem Soc 2011;133:13176-82.

22. Drepper T, Krauss U, Meyer zu Berstenhorst S, Pietruszka J, Jaeger K-E. Lights on and action! Controlling microbial gene expression by light. Appl Microbiol Biotechnol 2011;90:23-40.

23. van der Horst MA, Hellingwerf KJ. Photoreceptor proteins, "star actors of modern times": a review of the functional dynamics in the structure of representative members of six different photoreceptor families. Acc Chem Res 2004;37:13-20.

24. Shah S, Rangarajan S, Friedman SH. Light-activated RNA interference. Angew Chem Int Ed Engl 2005;44:1328-32.

25. Mikat V, Heckel A. Light-dependent RNA interference with nucleobase-caged siRNAs. Rna 2007;13:2341-7.

26. Jain PK, Shah S, Friedman SH. Patterning of gene expression using new photolabile groups applied to light activated RNAi. J Am Chem Soc 2011;133:440-6.

27. Ackroyd R, Kelty C, Brown N, Reed M. The history of photodetection and photodynamic therapy. Photochem Photobiol 2001;74:656-69.

28. Lee Y, Baron ED. Photodynamic therapy: current evidence and applications in dermatology. Semin Cutan Med Surg 2011;30:199-209.

29. Pervaiz S, Olivo M. Art and science of photodynamic therapy. Clin Exp Pharmacol Physiol 2006;33:551-6.

30. Dolmans DEJGJ, Fukumura D, Jain RK. TIMELINE: photodynamic therapy for cancer. Nat Rev Cancer 2003;3:380-7.

31. Christie JG, Kompella UB. Ophthalmic light sensitive nanocarrier systems. Drug Discov. Today 2008;13:124-34.

32. Triesscheijn M, Baas P, Schellens JHM, Stewart FA. Photodynamic therapy in oncology. The Oncologist 2006;11:1034-44.

33. Dougherty TJ, Henderson BW, Gomer CJ, Jori G, Kessel D, Korbelik M, et al. Photodynamic Therapy. J Natl Cancer Inst 1998;90:889-905.

34. Lai P-S, Lou P-J, Peng C-L, Pai C-L, Yen W-N, Huang M-Y, et al. Doxorubicin delivery by polyamidoamine dendrimer conjugation and photochemical internalization for cancer therapy. $\mathrm{J}$ Control Release 2007;122:39-46.

35. Lai P-S, Pai C-L, Peng C-L, Shieh M-J, Berg K, Lou P-J. Enhanced cytotoxicity of saporin by polyamidoamine dendrimer conjugation and photochemical internalization. J Biomed Mater Res 2008;87:147-55.

36. Lu H-L, Syu W-J, Nishiyama N, Kataoka K, Lai P-S. Dendrimer phthalocyanine-encapsulated polymeric micelle-mediated photochemical internalization extends the efficacy of photodynamic therapy and overcomes drug-resistance in vivo. J Control Release $2011 ; 155: 7-7$.

37. Nishiyama N, Arnida, Jang W-D, Date K, Miyata K, Kataoka K. Photochemical enhancement of transgene expression by polymeric micelles incorporating plasmid DNA and dendrimer-based photosensitizer. J Drug Target 2006;14:413-24.

38. Oliveira S, Fretz M, Høgset A, Storm G, Schiffelers RM. Photochemical internalization enhances silencing of epidermal growth factor receptor through improved endosomal escape of siRNA. BBA - Biomembranes 2007;1768:7-7.

39. Varkouhi AK, Schiffelers RM, van Steenbergen MJ, Lammers T, Hennink WE, Storm G. Photochemical internalization (PCI)-mediated enhancement of gene silencing efficiency of polymethacrylates and N,N,N-trimethylated chitosan (TMC) based siRNA polyplexes. J Control Release 2010;148:98-9.

40. Weyergang A, Selbo PK, Berstad MEB, Bostad M, Berg K. Photochemical internalization of tumor - targeted protein toxins. Lasers Surg Med 2011;43:721-33.

41. Kumaradas JC, Sherar MD. Edge-element based finite element analysis of microwave hyperthermia treatments for superficial tumours on the chest wall. Int J Hyperthermia 2003;19: 414-30.

42. Ohguri T, Imada H, Kato F, Yahara K, Morioka T, Nakano K, et al. Radiotherapy with $8 \mathrm{MHz}$ radiofrequency-capacitive regional hyperthermia for pain relief of unresectable and recurrent colorectal cancer. Int J Hyperthermia 2006;22:1-14.

43. Arthur RM, Straube WL, Trobaugh JW, Moros EG. Non-invasive estimation of hyperthermia temperatures with ultrasound. Int $\mathrm{J}$ Hyperthermia 2005;21:589-600.

44. Li J-L, Gu M. Gold-nanoparticle-enhanced cancer photothermal therapy. IEEE J Sel Top Quantum Electron 2010;16:989-96.

45. El-Sayed IH, Huang X, El-Sayed MA. Selective laser photothermal therapy of epithelial carcinoma using anti-EGFR antibody conjugated gold nanoparticles. Cancer Lett 2006; 239:7-7.

46. Kuo W-S, Chang Y-T, Cho K-C, Chiu K-C, Lien C-H, Yeh C-S, et al. Gold nanomaterials conjugated with indocyanine green for dual-modality photodynamic and photothermal therapy. Biomaterials 2012;33:3270-8.

47. O'Neal DP, Hirsch LR, Halas NJ, Payne JD, West JL. Photothermal tumor ablation in mice using near infrared-absorbing nanoparticles. Cancer Lett 2004;209:6-6.

48. Ruedas-Rama MJ, Walters JD, Orte A, Hall EAH. Fluorescent nanoparticles for intracellular sensing: a review. Anal Chim Acta 2012;751:1-23.

49. Delehanty JB, Susumu K, Manthe RL, Algar WR, Medintz IL. Active cellular sensing with quantum dots: transitioning from research tool to reality; a review. Anal Chim Acta 2012;750:63-81.

50. Dabbousi BO, Rodriguez-Viejo J, Mikulec FV, Heine JR, Mattoussi H, Ober R, et al. (CdSe) ZnS core-shell quantum dots: synthesis and characterization of a size series of highly luminescent nanocrystallites. J Phys Chem B 1997;101:9463-75.

51. Ji X, Zheng J, Xu J, Rastogi VK, Cheng TC, DeFrank JJ, et al. (CdSe) ZnS quantum dots and organophosphorus hydrolase bioconjugate as biosensors for detection of paraoxon. J Phys Chem B 2005;109:3793-9.

52. Chen Y, Rosenzweig Z. Luminescent CdS quantum dots as selective ion probes. Anal Chem 2002;74:5132-8.

53. Cordes DB, Gamsey S, Singaram B. Fluorescent quantum dots with boronic acid substituted viologens to sense glucose in aqueous solution. Angew Chem Int Ed Engl 2006;45: 3829-32.

54. Sandros MG, Gao D, Benson DE. A modular nanoparticle-based system for reagentless small molecule biosensing. J Am Chem Soc 2005;127:12198-9.

55. Shi L, De Paoli V, Rosenzweig N, Rosenzweig Z. Synthesis and application of quantum dots FRET-based protease sensors. J Am Chem Soc 2006;128:10378-9.

56. Zhu L, Zhu M-Q, Hurst JK, Li ADQ. Light-controlled molecular switches modulate nanocrystal fluorescence. J Am Chem Soc 2005;127:8968-70. 
57. Freeman R, Finder T, Bahshi L, Willner I. $\beta$-cyclodextrinmodified $\mathrm{CdSe} / \mathrm{ZnS}$ quantum dots for sensing and chiroselective analysis. Nano Lett 2009;9:2073-6.

58. Buck SM, Xu H, Brasuel M, Philbert MA, Kopelman R. Nanoscale probes encapsulated by biologically localized embedding (PEBBLEs) for ion sensing and imaging in live cells. Talanta 2004;63:41-59.

59. Lee Y-EK, Smith R, Kopelman R. Nanoparticle PEBBLE sensors in live cells and in vivo. Annu Rev Anal Chem (Palo Alto Calif) 2009;2:57-76.

60. Si D, Epstein T, Lee Y-EK, Kopelman R. Nanoparticle PEBBLE sensors for quantitative nanomolar imaging of intracellular free calcium ions. Anal Chem 2012;84:978-86.

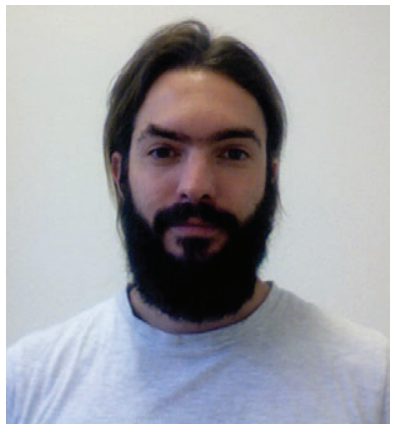

Roman Lehner is a 2nd year $\mathrm{PhD}$ student in the group of Prof. P. Hunziker, working within the SNF project "Intelligent Materials". Roman studied molecular biology at the University of Basel from where he received his MSc in structural biology in 2009. After his master diploma, he worked as a scientific associate at the Institute of Anatomy in Basel and for the spin-off company BioVersys. His work is focused on the design of a polymerbased drug delivery system, which can be functionalized to target particular tissue and combined with a stimulus responsiveness too light to explore activation of caged compounds.
61. Buck SM, Koo Y-EL, Park E, Xu H, Philbert MA, Brasuel MA, et al. Optochemical nanosensor PEBBLEs: photonic explorers for bioanalysis with biologically localized embedding. Curr Opin Chem Biol 2004;8:540-6.

62. Chen N, He Y, Su Y, Li X, Huang Q, Wang H, et al. The cytotoxicity of cadmium-based quantum dots. Biomaterials 2012;33:1238-44.

63. Skirtach AG, Antipov AA, Shchukin DG, Sukhorukov GB. Remote activation of capsules containing Ag nanoparticles and IR dye by laser light. Langmuir 2004;20:6988-92.

64. Lehner R, Wang X, Wolf M, Hunziker P. Designing switchable nanosystems for medical application. J Control Release 2012;161:307-16.

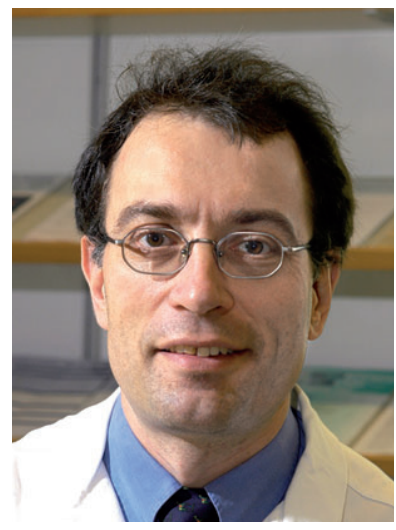

Patrick Hunziker has studied Medicine at the University of Zurich, Switzerland. He received a doctoral degree based on thesis work in experimental immunology from the University of Zurich and did further research in experimental hematology at University Hospital in Zurich, Switzerland. He earned specialist degrees in Internal Medicine, Cardiology and Intensive Care Medicine. As a fellow of the Massachusetts General Hospital, Harvard Medical School, he worked on cardiac imaging in a joint project with the Massachusetts Institute of Technology, Cambridge. His professional activities in Europe, the U.S., Africa and China gave him a broad insight into the needs for the medicine of the future in a variety of settings. Hunziker became involved in medical applications of Nanoscience in the late 1990s and has been the pioneer physician in Nanomedicine in Switzerland since then. With improved prevention, diagnosis and cure of cardiovascular disease as his main research topic, he worked in the nanoscience fields of atomic force microscopy, nano-optics, micro/nanofluidics, nanomechanical sensors and polymer nanocarriers for targeting. He is the founding president of the European Society of Nanomedicine, cofounder of the European Foundation for Clinical Nanomedicine and coinitiator of the European Conference for Clinical Nanomedicine and is clinically active as deputy head of the Clinic for Intensive Care Medicine at the University Hospital Basel, Switzerland. In November 2008 Patrick Hunziker became professor for Cardiology and Intensive Care Medicine at the University of Basel. 